DOI: $10.1515 / \mathrm{rrlm}-2017-0036$

\title{
FLT-3 ITD Positive Acute Basophilic Leukemia with Rare Complex Karyotype Presenting with Acute Respiratory Failure: Case Report
}

\author{
Ion Antohe ${ }^{1}$, Angela Dăscălescu ${ }^{1}$, Cătălin Dănăilă ${ }^{1}$, Mihaela Zlei², Iuliu Ivanov $^{3}$ \\ Adriana Sireteanu ${ }^{3}$, Oana Boca $^{3}$, Raluca Oană ${ }^{4}$, Petru Cianga ${ }^{5 *}$ \\ 1. Hematology Department, "Grigore T. Popa” University of Medicine and Pharmacy Iaşi, Romania \\ Hematology Department, Regional Oncology Institute, Iaşi, Romania \\ 2. Immunophenotyping Department, Regional Oncology Institute, Iaşi, Romania \\ 3. Molecular Diagnostic Department, Regional Oncology Institute, Iaşi, Romania \\ 4. Cytology Department, Regional Oncology Institute, Iaşi, Romania
}

5. Immunology Department, "Grigore T. Popa” University of Medicine and Pharmacy, Iaşi, Romania

\begin{abstract}
Background: Acute basophilic leukemia is a rare subtype of acute myeloid leukemia, as categorized by the 2008 World Health Organization classification of myeloid neoplasms. Acute basophilic leukemia diagnosis requires thorough morphological, cytochemical, immunophenotypic, molecular, and cytogenetic studies and exclusion of other hematological neoplasms associating basophilia.

The disease course is defined by histamine driven, occasionally life-threatening respiratory, cardiovascular, cutaneous or digestive complications, as well as primary refractoriness to standard therapy.

Clinical presentation: We herein report a case of a 63-year-old asthmatic female patient diagnosed with acute basophilic leukemia, associated with previously unpublished cytogenetic features and FLT-3 ITD mutation, pulmonary leukostasis and spontaneous pulmonary capillary leak syndrome, which worsened immediately following chemotherapy initiation. Respiratory complications were successfully managed, but recrudesced upon emergence of refractory disease and were ultimately fatal.

We highlight the likelihood of pulmonary complications induced by basophil degranulation and tumor lysis in hypercellular acute basophilic leukemia and the potential benefit of histamine receptor blockade in this setting.
\end{abstract}

Keywords: Acute Basophilic Leukemia, FLT-3 ITD, leukostasis, acute respiratory failure

Received: 13 th August 2017; Accepted: 26 th October 2017; Published: $15^{\text {th }}$ December 2017

*Corresponding author: Petru Cianga, "Grigore T. Popa” University of Medicine and Pharmacy Iaşi, Romania. E-mail: petrucianga@hotmail.com 


\section{Introduction}

Acute Basophilic Leukemia is an uncommon hematological malignancy, currently categorized by the 2008 World Health Organization (WHO) classification as a particular subtype of unspecified acute myeloid leukemia (AML), often associated with an aggressive clinical course and dismal prognosis [1]. Complications related to high histamine release, including skin rash, peptic ulcer, gastrointestinal bleeding, anaphylaxis and respiratory symptoms have been reported $[2,3]$. Meticulous morphological, cytochemical, immunophenotypic, molecular, and cytogenetic studies are required to fully characterize acute basophilic leukemia. Differential diagnosis includes other myeloid neoplasms associated with basophilia, namely AML with $\mathrm{t}(6 ; 9)(\mathrm{p} 23 ; \mathrm{q} 14)$, acute promyelocytic leukemia with basophilic differentiation, blastic transformation of chronic myelogenous leukemia, primary myelofibrosis, or rare acute lymphoblastic leukemia cases with prominent cytoplasmic granules $[1,4]$. Noteworthy, even though multiple chromosomal abnormalities have been reported involving chromosomes $1,2,6,7,8,11,12,16,17,19,21$ and $\mathrm{X}$, no recurrent cytogenetic or molecular anomalies have been described in acute basophilic leukemia patients [3,5-13]. Acute basophilic leukemia management remains poorly defined, relying on cytarabine and anthracycline-based induction regimens, palliative cytoreductive treatment, targeted therapies (tyrosine kinase inhibitors) or allogeneic stem cell transplant in selected cases $[7,14]$. Shortened disease free survival (DFS) or refractory disease are common among acute basophilic leukemia patients $[7,14]$.

\section{Case report:}

A 63-year-old female patient with a medical history of diabetes, hypertension, bronchial asthma since the age of 35 , and diagnosed with non-Hodgkin lymphoma at age 36 , for which she had previously received chemo- and radiotherapy, was admitted for fatigue and exertional dyspnea. Physical examination was unremarkable, with no cutaneous lesions documented. Asthma control had been previously achieved with inhaled long acting bronchodilators and corticosteroids, with no identifiable exacerbations within the 12 months preceding admission to our clinic.

Laboratory findings revealed a hemoglobin level of $6.7 \mathrm{~g} / \mathrm{dl}, 66 \times 10^{9} / \mathrm{L}$ platelets, $18 \times 10^{9} / \mathrm{L}$ leukocytes, $1.2 \times 10^{9} / \mathrm{L}$ neutrophils, $0.9 \times 10^{9} / \mathrm{L}$ basophils and $68 \%$ agranular blasts with basophilic cytoplasm. Bone marrow (BM) investigation revealed hypercellularity with $70 \%$ infiltration
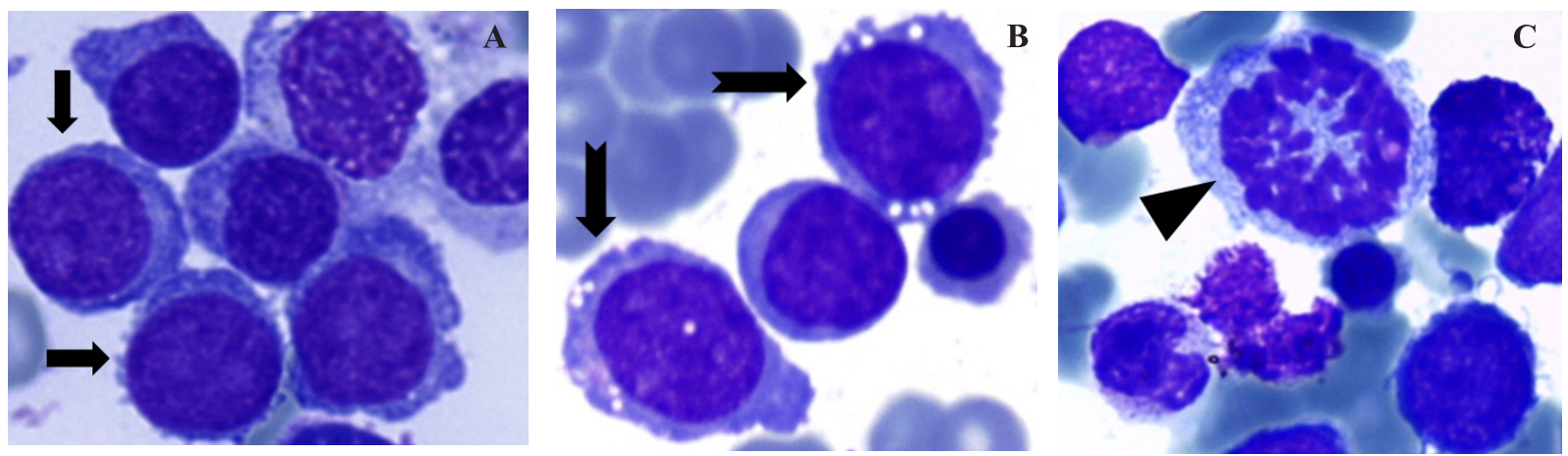

Figure 1. Bone marrow infiltration with medium sized agranular blasts, with fine chromatin, basophilic cytoplasm and proerythroblast (A, dark arrow) or Burkitt-like (B, notched dark arrow) appearance. Frequent karyorrhexis was present as a feature of erythroid dysplasia $(C$, dark arrowhead) (May Grünwald Giemsa, x100). 
with ambiguous lineage agranular blasts, some resembling proerythroblasts and others with cytoplasmic vacuoles. The following features of bone marrow dysplasia were present in $10 \%$ of the examined nucleated cells: neutrophil hypogranularity, nuclear erythroid dysplasia and megakaryocyte hypolobulation (Figure 1).

Flow cytometry immunophenotyping confirmed the basophilic nature of the blasts and also revealed the presence of a minor subclone with early mastocyte lineage commitment (Table 1) (Figure 2).

Molecular testing for FLT-3 ITD (FMS-like tyrosine kinase receptor-3-internal tandem duplication) was positive. CBFB-MYH11, RUNX1RUNX1T1, PML-RAR $\alpha$, FLT3 D835 mutation, and NPM 1 were negative. Conventional cytogenetic examination using GTG banding revealed a complex karyotype, with multiple numerical and structural aberrations (14 metaphases with structural anomalies consisting of $\mathrm{t}(1 ; 4)$ (p35;q35), del(5)(q31->qter), t(5;20)(p15;p13) and 17 metaphases with numeric anomalies: 11 metaphases with hyperdiploidy (47-50 chromosomes) and 6 metaphases with hypodiploidy. $\mathrm{t}(9,22)$ or $\mathrm{t}(6,9)$ were not identified. $\operatorname{del}(5)(\mathrm{q} 31$ $>$ qter) was confirmed by interphase fluorescence in situ hybridization (FISH) testing (Figure 3). No 11q23 rearrangements were described.
A diagnosis of therapy related FLT-3-ITD positive acute basophilic leukemia was established, based on the medullary infiltrate phenotype and on the cytogenetic and molecular exclusion of other basophilia-associating myeloproliferative syndromes. Ultrastructural characterization of basophilic blasts by electron microscopy was not available.

Within 72 hours following admission, the patient developed symptoms of progressive respiratory failure, with bronchospasm and wheezing. In parallel, a threefold increase of leukocytes $\left(47 \times 10^{\circ} \mathrm{L}\right)$ was recorded. The patient remained afebrile and $\mathrm{C}$ reactive protein was only mildly increased $(10 \mathrm{mg} / \mathrm{L}$, normal range: 0 to $1 \mathrm{mg} / \mathrm{L}$ ), underlining the unlikelihood of pulmonary infection. Thoracic computed tomography revealed bilateral ground glass infiltrates and ruled out pulmonary embolism.

Furthermore, broad spectrum antibiotherapy and a short empirical course of Voriconazole led to no respiratory improvement. Taking into account the low probability of pulmonary infection and the temporal concurrence of progressive leukocytosis and respiratory failure with bronchospastic features in a patient with previously controlled asthma, we considered the diagnosis of non-cardiogenic pulmonary edema secondary to pulmonary leukostasis. Normal serum hista-

Table 1. Expression pattern of most relevant markers evaluated by flow cytometry

\begin{tabular}{|l|c|c|c|c|c|c|c|c|c|}
\hline \multirow{2}{*}{$\begin{array}{l}\text { Cell } \\
\text { populations }\end{array}$} & \multicolumn{9}{|c|}{ Relevant markers } \\
\cline { 2 - 10 } & CD34 & CD117 & HLA/DR & CD203c & CD22 & MPO & CD13 & CD33 & CD56 \\
\hline $\begin{array}{l}\text { AML clone 1 } \\
(72 \%)\end{array}$ & + & + & $+/-$ & $-/+$ & + & - & + & + & + \\
\hline $\begin{array}{l}\text { AML clone 2 } \\
(2,5 \%)\end{array}$ & + & ++ & - & $-/+$ & - & - & + & - & - \\
\hline
\end{tabular}

AML clone 1= cells with early basophil lineage commitment, containing 4\% more mature (phenotypically related) basophils AML clone $2=$ cells with early mast cell lineage commitment

$+=$ positive

- = negative

$-/+=$ partial expression, mostly negative

$+/-=$ partial expression, mostly positive.

Grey fields $=$ most relevant markers for basophil/mastocyte lineage assignment. Percentages are calculated from the whole sample. 

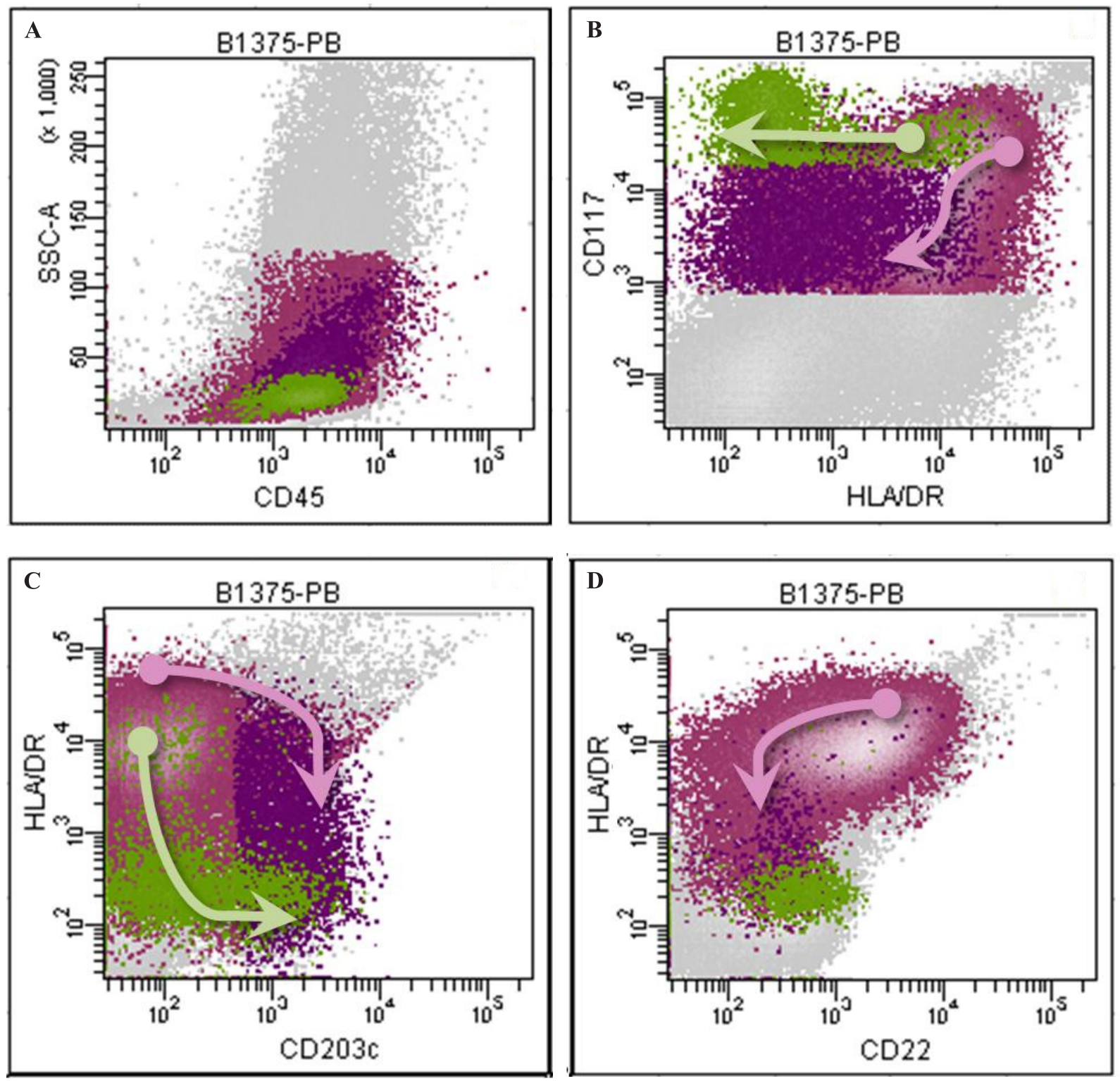

Figure 2. Expression pattern of most relevant markers evaluated by flow cytometry.

Two AML clones were identified by flow cytometry, using an 8 color 25 antibodies panel (A: side-scatter vs CD45). A major AML clone (72\%, magenta) was assigned to cells with early basophil lineage commitment, also containing $4 \%$ more mature basophils (magenta dark). A second, minor (2.5\%) malignant clone represents precursors with early mast cell lineage commitment (green). Arrows highlight a continuous expression pattern (B: CD117 vs HLA-DR; C: HLA-DR vs CD203c; D: HLA-DR vs CD22) between different clones, suggestive for their lineage connection. 


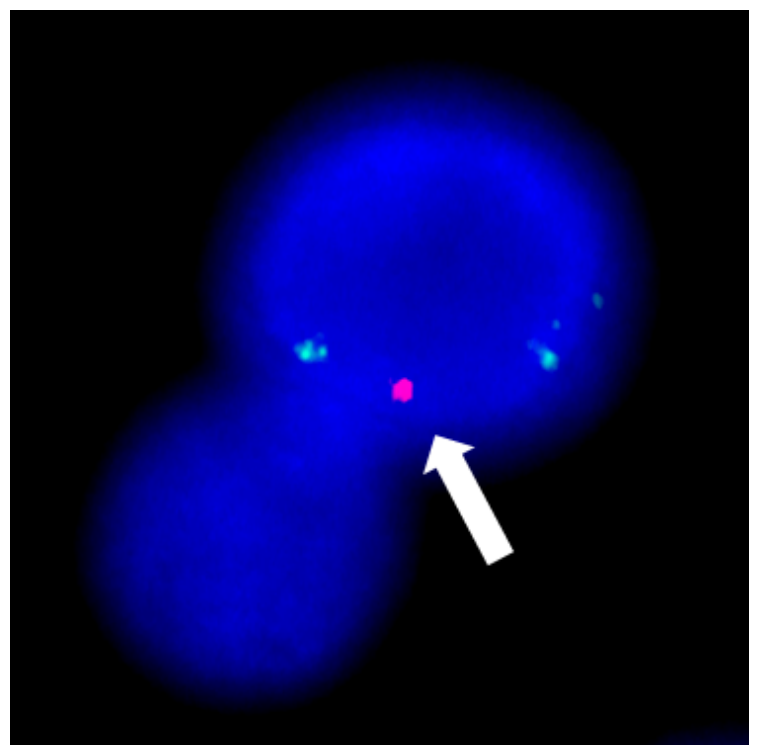

Figure 3. del(5q31) (white arrow) (LSI EGR1/

D5S23,D5S721 Dual Color Probe - Vysis)interphasic fluorescence in situ hybridization

(FISH).

mine and tryptase levels were considered unreliable, as they were measured after corticotherapy initiation.

Consequently, standard " $3+7$ " (Idarubicin $12 \mathrm{mg} / \mathrm{m}^{2}$ intravenously for three days and cytarabine $100 \mathrm{mg} / \mathrm{m}^{2}$ in continuous intravenous infusion for seven days) induction therapy was initiated. Within one hour after chemotherapy initiation the patient presented grade 2 cytokine release syndrome (hypotension, fever, chills, profuse sweats, rash and bronchospasm exacerbation).

Under chemotherapy and intravenous methylprednisolone, bronchodilators and histamine receptors 1 and 2 blockade the respiratory symptoms and bronchospasm gradually improved over the next 72 hours.

On day +18 post induction, the hemogram showed $7.2 \times 10^{9} / \mathrm{L}$ leukocytes and peripheral blood smear examination revealed blast cells, attesting refractory disease. Within 24 hours the respiratory symptoms present on diagnosis reoc- curred. A re-induction course of $500 \mathrm{mg}$ cytarabine for three days was attempted, but the patient died due to progressive respiratory failure.

\section{Discussion}

Acute basophilic leukemia remains a poorly defined entity, due to lack of standardized diagnostic criteria, treatment regimens, and scarcity of case reports. According to the $2008 \mathrm{WHO}$ classification [1] and its 2016 revision [15], acute basophilic leukemia diagnosis requires specific morphological and immunophenotypic criteria and exclusion of other hematological basophilia-associating neoplasms. No characteristic recurrent cytogenetic or molecular abnormalities have been defined to date.

It should be noted that some acute basophilic leukemia cases reported before 2008 do not fit the current WHO definition and represent in fact blastic phases of chronic myeloid leukemia presenting with significant basophilia [16].

FLT-3-ITD mutation occurs in about $30 \%$ of adult AML cases and it is correlated with reduced DFS and overall survival [17,18]. FLT3-ITD mutation is a common finding in AML patients with $\mathrm{t}(6: 9)(\mathrm{p} 23: \mathrm{q} 34)$ (78\% of cases) [1], alongside BM dysplasia and a complex karyotype. In our case, the diagnosis of acute basophilic leukemia with mutated FLT3-ITD is strongly supported by flow cytometry data and cytogenetic studies. Furthermore, to the best of our knowledge, this is the first reported case of FLT3-ITD positive acute basophilic leukemia, doubled by a complex, also previously unreported karyotype.

Additionally, the patient's history of chemoand radio-treated non Hodgkin lymphoma and multilineage BM dysplastic features suggest a possible preceding myelodysplastic syndrome. Although BM dysplastic features were present, they did not surpass the $50 \%$ threshold necessary for the diagnosis of AML with myelodysplasia-related features. Despite the late onset of the 
disease relative to the previous lymphoma-targeted chemotherapy, the presence of chromosome 5 anomalies is suggestive for therapy-related acute basophilic leukemia.

Furthermore, this case features a rare presentation of hypercellular acute basophilic leukemia precipitating phenomena of respiratory failure in a patient with previously controlled asthma.

The extent to which asthma history might have contributed to a leukemia phenotype shift towards the basophil lineage is difficult to evaluate. Literature cites a case of mixed lineage eosinophil-basophil acute leukemia in a young female patient associating asthma [19]. T helper 2 lymphocytes trafficking from airways of asthmatic patients to the BM apparently induce up-regulation of interleukin-5 (IL-5) receptors on CD34+ progenitors, thus altering normal hematopoiesis and favoring IL-5 mediated expansion of eosinophil and basophil precursors [20]. However, it is virtually impossible to assess whether IL-5-induced modifications in the BM microenvironment of asthma patients might contribute to leukemogenesis and the subsequent basophilic and mastocytic differentiation in this particular case.

The temporal concurrence of respiratory symptoms and progressive leukocytosis strongly suggest intricate phenomena of pulmonary leukostasis and capillary leak syndrome. Ground glass opacities sparing the lung bases and the subpleural space are evocative of non-cardiogenic pulmonary edema. Pulmonary leukostasis requires endothelial cell activation and expression of various adhesion molecules by the leukemic blasts, including CD56, irrespective of the absolute leukocyte count $[21,22]$. In this case, CD56 expression on basophilic blasts might have specifically facilitated the development of leukostasis. Spontaneous or post therapeutic worsening of respiratory failure in patients with pulmonary leukostasis has been previously cited in hypercellular myelomonocytic leukemias [23,24].
Moreover, bronchospasmexacerbationalongside hypotension and rash following chemotherapy initiation strongly suggest the implication of vaso- and broncho-active mediators released upon lysis of basophilic blast cells. Pulmonary damage in myelomonocytic leukemias associating eosinophilia has been linked to eosinophil degranulation $[25,26]$, emphasizing the likelihood of a similar mechanism in acute basophilic leukemia. Consequently, steroid premedication and double histamine receptor blockade was initiated and succeeded to limit the impact of histamine related complications. Ultimately, reappearance of respiratory distress upon emergence of refractory disease and subsequent basophil proliferation further indicate the role of tumor progression and cytokine release in the pathogenesis of pulmonary edema and leukostasis.

Previously published data have certified the existence of certain clonal relationship between myeloid blasts and BM mast cells in an AML patient [27]. The presence of two different AML clones, identified by differential expression of HLA-DR, CD117 and CD203c suggest a putative common origin of these subclones, i.e. a common malignant basophil-mastocyte progenitor, even though the existence of its normal counterpart in human hematopoiesis has yet to be demonstrated [28].

\section{Conclusion}

Acute basophilic leukemia is a very rare AML subtype whose diagnosis poses a real challenge. Furthermore, acute basophilic leukemia management is still unstandardized and one should always be aware of the potential of the disease to lead to life-threatening histamine-driven pulmonary, cardiovascular, digestive, and skin complications. Spontaneous or post chemotherapy respiratory distress should raise suspicion of pulmonary capillary leak induced by basophil lysis or degranulation. Dou- 
ble histamine receptor blockade, steroid therapy and chemotherapy initiation are recommended in such cases, as well as empirical antibiotherapy, since infection cannot be definitely ruled out.

Noteworthy, normal serum levels of histamine and tryptase should not discourage therapy in the setting of high clinical suspicion of histamine-driven complications.

\section{Acknowledgments}

We are particularly indebted to Professor Alberto Orfao for valuable advice in interpreting flow cytometry data.

\section{Conflicts of interest}

The authors report no conflicts of interest.

\section{List of abbreviations}

$\mathrm{AML}=$ acute myeloid leukemia

$\mathrm{BM}=$ bone marrow

CBFB-MYH11= core binding factor beta- myosine heavy chain 11

$\mathrm{CD}=$ cluster of differentiation

$\mathrm{FISH}=$ fluorescence in situ hybridization

FLT-3 ITD= FMS-like tyrosine kinase receptor-3- internal tandem duplication

Il-5= interleukin 5

HLA-DR = human leukocyte antigen DR

NPM 1= nucleophosmin 1

PML-RAR $\alpha=$ promyelocytic leukemia/retinoic acid receptor $\alpha$

RUNX1-RUNX1T1 = runt-related transcription factor 1-runt-related transcription factor 1 target 1

$\mathrm{WHO}=$ World Health Organization

\section{References}

1. Arber DA, Brunning RD, Orazi A, Porwit A, Peterson L, Thiele J, et al. Acute Basophilic Leukemia. In: Swerdlow SH, Campo E, Harris NL, et al., editors. WHO Classification of Tumours of Haematopoietic and Lymphoid Tissues. Lyon (Fr): IARC Press; 2008. p 137-8.

2. Luo X-H, Zhu Y, Tang X-Q. Acute basophilic leukemia presenting with maculopapular rashes and a gastric ulcer: A case report. Oncol Lett. 2014;8(6):2513-6. DOI: 10.3892/ol.2014.2544

3. Bernini JC, Timmons CF, Sandler ES. Acute basophilic leukemia in a child. Anaphylactoid reaction and coagulopathy secondary to vincristine-mediated degranulation. Cancer. 1995;75:110-4. DOI: 10.1002/1097-0142(19950101)75:1<110::AID-CNCR2820750118>3.0.CO;2-I

4. Kritharis A, Brody J, Koduru P, Teichberg S, Allen SL. Acute basophilic leukemia associated with loss of gene ETV6 and protean complications. J Clin Oncol. 2011;29(21):623-6. DOI: 10.1200/JCO.2010.34.5710

5. Pidala J, Pinilla-Ibarz J, Cualing HD. A case of acute basophilic leukemia arising from chronic myelogenous leukemia with development of $\mathrm{t}(7 ; 8)(\mathrm{q} 32 ; \mathrm{q} 13)$. Cancer Genet Cytogenet. 2008;182(1):46-9. DOI: 10.1016/j. cancergencyto.2007.12.009

6. Eveillard M, Desjonqueres A, de Nantes C. Acute basophilic leukemia. Blood. 2014;123(20):3071. DOI: 10.1182/blood-2014-02-555698

7. Shvidel L, Shaft D, Stark B, Shtalrid M, Berrebi A, Resnitzky P. Acute basophilic leukaemia: Eight unsuspected new cases diagnosed by electron microscopy. Br J Haematol. 2003;120(5):774-81. DOI: 10.1046/j.13652141.2003.04167.x

8. Zhao Y, Kong D, Han L, Hu LH, Zhang Z, Liu JJ, et al. Arsenic trioxide in treatment of de novo acute basophilic leukemia. Chin Med J. 2013;126(3):593-4.

9. Dastugue N, Duchayne E, Kuhlein E, Rubie H, Demur C, Aurich J, et al. Acute basophilic leukaemia and translocation $\mathrm{t}(\mathrm{X} ; 6)(\mathrm{p} 11 ; \mathrm{q} 23)$. Br J Haematol. 1997;98(1):170-6. DOI: $10.1046 /$ j.13652141.1997.1562968.x

10. Seth T, Vora A, Bhutani M, Ganessan K, Jain P, Kochupillai V. Acute basophilic leukemia with $\mathrm{t}(8 ; 21)$. Leuk Lymphoma. 2004;45(3):605-8. DOI: $10.1080 / 10428190310001598053$

11. Kokandakar H, Tembhare P, Mamoon A, Mulay VM, Bhople KS. Acute basophilic leukaemia: A case report. Indian J Pathol Microbiol. 2007;50(2):443-6.

12. Bahmanyar $M$, Chang $H$. Acute myeloid leukemia with myelodysplasia-related changes demonstrating prominent basophilic differentiation. Blood. 2016;127(20):2503. DOI: 10.1182/ blood-2016-02-701144

13. Giagounidis A, Hildebrandt B, Heinsch M, Germing U, Aivado M, Aul C. Acute basophilic leukemia. Eur J Haematol. 2001;67(2):72-6. DOI: 10.1034/j.16000609.2001.t01-1-00487.x

14. Valent P, Sotlar K, Blatt K, Hartmann K, Reiter A, Sadovnik I, et al. Proposed diagnostic criteria and 
classification of basophilic leukemias and related disorders. Leukemia. 2017;31(4):788-97. DOI: 10.1038/ leu. 2017.15

15. Arber DA, Orazi A, Hasserjian R, Thiele J, Borowitz MJ, Le Beau MM, et al. The 2016 revision to the World Health Organization classification of myeloid neoplasms and acute leukemia. Blood.2016;127(20):2391 -405. DOI: 10.1182/blood-2016-03-643544

16. Staal-Viliare A, Latger-Cannard V, Rault J, Didion J, Grégoire MJ, Bologna S, et al. À propos d'un cas de leucémie à basophiles de novo: Critères diagnostiques et revue de la littérature. Ann Biol Clin. 2006;64(4):3615.

17. Kottaridis PD, Gale RE, Frew ME, Harrison G, Langabeer SE, Belton AA, et al. The presence of a FLT3 internal tandem duplication in patients with acute myeloid leukemia (AML) adds important prognostic information to cytogenetic risk group and response to the first cycle of chemotherapy: Analysis of 854 patients from the United Kingdom medical research council AML 10 and 12 trials. Blood. 2001;98(6):1752-9. DOI: 10.1182/ blood.V98.6.1752

18. Schnittger S, Schoch C, Dugas M, Kern W, Staib P, Wuchter C, et al. Analysis of FLT3 length mutations in 1003 patients with acute myeloid leukemia: Correlation to cytogenetics, FAB subtype, and prognosis in the AMLCG study and usefulness as a marker for the detection of minimal residual disease. Blood. 2002;100(1):59-66. DOI: 10.1182/blood.V100.1.59

19. Shvidel L, Sigler E, Shtalrid M, Berrebi A, Resnitzky P. Hybrid eosinophilic-basophilic acute myeloid leukaemia diagnosed by electron microscopy. $\mathrm{Br} \mathrm{J}$ Haematol. 2007;137(4):381-3. DOI: 10.1111/j.13652141.2007.06579.x

20. Gauvreau GM, Ellis AK, Denburg JA. Haemopoietic processes in allergic disease: Eosinophil/basophil development. Clin Exp Allergy. 2009;39(9):1297306. DOI: 10.1111/j.1365-2222.2009.03325.x

21. Porcu P, Cripe LD, Nq EW, Bhatia S, Danielson CM, Orazi A, et al. Hyperleukocytic Leukemias and Leu- kostasis: A review of pathophysiology, clinical presentation and management. Leuk Lymphoma. 2000;39(12):1-18. DOI: $10.3109 / 10428190009053534$

22. Novotny JR, Nuckel H, Duhrsen U. Correlation between expression of CD56/NCAM and severe leukostasis in hyperleukocytic acute myelomonocytic leukaemia. Eur J Haematol. 2006;76(4):299-308. DOI: 10.1111/j.1600-0609.2005.00607.x

23. Azoulay É, Fieux F, Moreau D, Thiery G, Rousselot P, Parrot A, et al. Acute Monocytic leukemia presenting as acute respiratory failure. Am J Respir Crit Care Med. 2003;167(10):1329-33. DOI: $10.1164 /$ rccm.200206-554OC

24. Myers TJ, Cole SR, Klatsky AU, Hild DH. Respiratory failure due to pulmonary leukostasis following chemotherapy of acute nonlymphocytic leukemia. Cancer. 1983;51(10):1808-13. DOI: 10.1002/1097-0142(19830515)51:10<1808::AID-CNCR2820511009>3.0.CO;2-Y

25. Dombret H, Hunault M, Faucher C, Dombret MC, Degos L. Acute lysis pneumopathy after chemotherapy for acute myelomonocytic leukemia with abnormal marrow eosinophils. Cancer. 1992;69:1356-61. DOI: 10.1002/1097-0142(19920315)69:6<1356::AID-CNCR2820690609>3.0.CO;2-B

26. Lester WA, Hull DR, Fegan CD, Morris TC. Respiratory failure during induction chemotherapy for acute myelomonocytic leukaemia (FAB M4Eo) with Ara-C and all-trans retinoic acid. Br J Haematol. 2000;109(4):84750. DOI: 10.1046/j.1365-2141.2000.02043.x

27. Frederiksen JK, Shao L, Bixby DL, Ross CW. Shared clonal cytogenetic abnormalities in aberrant mast cells and leukemic myeloid blasts detected by single nucleotide polymorphism microarray-based whole-genome scanning. Gene Chromosome Canc. 2016;55(4):38996.DOI: $10.1002 /$ gcc. 22342

28. Arock M, Schneider E, Boissan M, Tricottet V, Dy M. Differentiation of human basophils: An overview of recent advances and pending questions. J Leukoc Biol. 2002;71(4):557-64. 